\title{
BMJ Open Effect of whole-body vibration exercise in preventing falls and fractures: a systematic review and meta-analysis
}

\author{
Ditte Beck Jepsen, ${ }^{1,2}$ Katja Thomsen, ${ }^{2,3}$ Stinus Hansen,, ${ }^{2,4}$ Niklas Rye Jørgensen, ,,6 \\ Tahir Masud, , ,2,7 Jesper Ryg ${ }^{1,2}$
}

To cite: Jepsen DB, Thomsen K, Hansen $\mathrm{S}$, et al. Effect of whole-body vibration exercise in preventing falls and fractures: a systematic review and meta-analysis. BMJ Open 2017;7:e018342. doi:10.1136/ bmjopen-2017-018342

- Prepublication history and additional material for this paper are available online. To view these files, please visit the journal online (http://dx.doi. org/10.1136/bmjopen-2017018342).

Received 21 June 2017

Revised 27 September 2017

Accepted 10 November 2017

CrossMark

${ }^{1}$ Department of Geriatric Medicine, Odense University Hospital, Odense, Denmark

${ }^{2}$ Institute of Clinical Research, University of Southern Denmark, Odense, Denmark

${ }^{3}$ Department of Geriatric Medicine, Odense University Hospital, Svendborg, Denmark ${ }^{4}$ Department of Endocrinology, Odense University Hospital,

Odense, Denmark

${ }^{5}$ Department of Clinical Biochemistry, Rigshospitalet, Copenhagen, Denmark ${ }^{6} \mathrm{OPEN}$-Odense Patient data Explorative Network, Odense University Hospital/ University of Southern Denmark, Odense, Denmark

${ }^{7}$ Department of Geriatric Medicine, Nottingham University Hospitals NHS Trust, Nottingham, UK

Correspondence to MD Ditte Beck Jepsen; ditte.beck.jepsen2@rsyd.dk

\section{ABSTRACT}

Objective To investigate the effect of whole-body vibration exercise (WBV) on fracture risk in adults $\geq 50$ years of age.

Design A systematic review and meta-analysis calculating relative risk ratios, fall rate ratio and absolute weighted mean difference using random effects models. Heterogeneity was estimated using $\mathrm{I}^{2}$ statistics, and the Cochrane Collaboration's risk of bias tool and the GRADE approach were used to evaluate quality of evidence and summarise conclusions.

Data sources The databases PubMed, Embase and the Cochrane Central Register from inception to April 2016 and reference lists of retrieved publications.

Eligibility criteria for selecting studies Randomised controlled trials examining the effect of WBV on fracture risk in adults $\geq 50$ years of age. The primary outcomes were fractures, fall rates and the proportion of participants who fell. Secondary outcomes were bone mineral density (BMD), bone microarchitecture, bone turnover markers and calcaneal broadband attenuation (BUA).

Results 15 papers (14 trials) met the inclusion criteria. Only one study had fracture data reporting a nonsignificant fracture reduction (risk ratio $(\mathrm{RR})=0.47,95 \% \mathrm{Cl}$ 0.14 to $1.57, P=0.22$ ) (moderate quality of evidence). Four studies $(n=746)$ showed that WBV reduced the rate of falls with a rate ratio of $0.67(95 \% \mathrm{Cl} 0.50$ to $0.89, \mathrm{P}=0.0006$; $\mathrm{I}^{2}=19 \%$ ) (moderate quality of evidence). Furthermore, data from three studies $(n=805)$ found a trend towards falls reduction $\left(\mathrm{RR}=0.76,95 \% \mathrm{Cl} 0.48\right.$ to $1.20, \mathrm{P}=0.24 ; \mathrm{l}^{2}=24 \%$ ) (low quality of evidence). Finally, moderate to low quality of evidence showed no overall effect on BMD and only sparse data were available regarding microarchitecture parameters, bone turnover markers and BUA.

Conclusions WBV reduces fall rate but seems to have no overall effect on BMD or microarchitecture. The impact of WBV on fractures requires further larger adequately powered studies. This meta-analysis suggests that WBV may prevent fractures by reducing falls.

PROSPERO registration number CRD42016036320; Pre-results.

\section{INTRODUCTION}

Fragility fractures are associated with much morbidity, mortality and cost to society. ${ }^{12}$ In Europe, the direct medical cost of these fractures has been estimated at $€ 31.7$ billion

\section{Strengths and limitations of this study}

This is the first systematic review comprehensively conducting a meta-analysis on the effect of wholebody vibration exercise (WBV) on the overall risk of fractures, including falls.

- An extensive systematic literature search identified all available randomised controlled trials using WBV in adults aged 50 on falls, fractures and bone parameters.

- A risk of selection bias exists due to no inclusion of non-English language literature, grey literature or adverse effects.

per year, expected to rise to $€ 76.7$ billion by 2050. ${ }^{3}$ Propensity to fall and osteoporosis are the major determinants of fragility fractures. $^{145}$

One-third of the population over 65 years of age falls at least once a year. ${ }^{6}$ Increasing age, frailty, comorbidity, reduced muscle strength and impaired balance contribute to the risk of falls. ${ }^{46}$ In Europe, 22 million women and 5.5 million men were estimated to have osteoporosis in 2010. ${ }^{1}$ WHO criteria for diagnosing osteoporosis is based on measurement of bone mineral density (BMD), but there are also other important aspects of bone fragility including microarchitecture and bone turnover. ${ }^{78}$ The combination of age-related bone loss and an increased risk of falls cause a higher incidence of fragility fractures in people aged 50 years or more. ${ }^{19}$ With an ageing population, the increased cost caused by fragility fractures poses a significant challenge to healthcare systems. ${ }^{13}$ Reducing fracture risk with the dual approach of lowering fall risk and enhancing bone strength is therefore desirable. ${ }^{10}$

Whole-body vibration exercise (WBV) has been proposed as an exercise modality anabolic to bone, capable of enhancing balance and improving muscle strength. ${ }^{11-14}$ Animal studies have showed that mechanical 
signals introduced via vibration stimulate bone formation and suppress bone resorption. ${ }^{15-17}$ The accelerations from vibration platforms are transmitted to the person standing on the plate from the feet to the adjacent muscles and bones. When the plate moves the adjacent muscles provide contractions as a reflex to the stimulus. ${ }^{18}$ WBV has been proposed to counteract ageing's suppression on osteoblast activity thereby preventing bone loss. ${ }^{15}$ WBV with high magnitude (high frequency and/or amplitude) has shown to increase muscular activation, and this technique has been suggested as an alternative to weight-bearing exercise. ${ }^{18} \mathrm{WBV}$ training protocols varies from a few minutes vibration up to 20 min depending on the peak acceleration.

The vibration plates can be assessed at home, in the local community or at rehabilitation units with different forms of monitoring and supervision. The WBV is used as an intervention aimed at preventing bone loss, enhancing muscle strength and balance.

Several studies have investigated the role of WBV on BMD, muscle strength and balance. ${ }^{11} 1214$ 19-24 However, the results have been inconsistent perhaps due to differences in types of vibration studied: intervention designs, populations assessed and study quality. Fewer studies have focused on the effect of WBV on falls and bone strength parameters other than BMD. ${ }^{19} 20$ 25-27

Previous systematic reviews on the effect of WBV on balance and muscle strength in older adults have reported improvement in lower extremity muscle strength or in certain balance measures. ${ }^{28-32}$ Systematic reviews focusing on BMD have shown inconsistent results, ${ }^{32-35}$ with some showing no overall effect, ${ }^{32}$ others a small increase in BMD of the hip ${ }^{33}$ or no effect on the hip but an effect on lumbar spine, ${ }^{35}$ while some found a BMD increase in certain subgroups only. ${ }^{34}$ To the best of our knowledge, no systematic review has comprehensively investigated the role of WBV on fragility fractures and overall risk of fragility fractures, including falls and bone quality.

The objectives of this systematic review were to address if WBV in adults over 50 years of age could affect the incidence of fractures, falls, as well as estimates of bone mass, architecture and turnover.

\section{METHODS}

\section{Data sources and searches}

Literature searches were conducted in the following electronic bibliographic databases: PubMed, Embase and The Cochrane Library (Cochrane Central Register of Controlled Trials). The searches were conducted from inception to 4 April 2016. Additionally, we performed manual searches of the reference lists of retrieved publications and earlier reviews. ${ }^{29}{ }^{32-35}$ An updated search was conducted by end of January 2017 to check for any new relevant studies prior to submission.

The search string was structured with librarian assistance using the PICO method: $\mathrm{P}$ (population)=adults $\geq 50$ years of age, I (intervention) $=$ whole-body sinusoidal vibration (ie, constant vibration frequency) from a platform, C (comparison)=no intervention, sham, normal care or same exercise in both arms and $\mathrm{O}$ (outcome)=fractures, falls and bone property parameters.

The searches were conducted without filters or restrictions, and the search string is available in online supplementary appendix 1 .

\section{Study selection}

One author (DBJ) screened title and abstracts. Two authors (DBJ, KT) independently evaluated the full-text papers and eligibility. Conflicts were resolved by a third author (JR). The selection was conducted using the software Covidence (Covidence systematic review software; Veritas Health Innovation, Melbourne, Australia) and a standardised eligibility form.

Inclusion criteria: randomised controlled trials (RCTs) investigating the effect of WBV on fractures, falls and bone properties within the population $\geq 50$ years of age. WBV had to be whole-body sinusoidal vibration (ie, constant vibration frequency) from a platform that vibrates vertically or side alternating, with no restriction on frequency, amplitude or magnitude. The participants had to stand during the WBV. The control groups had to have either no intervention, usual care, sham vibration, activity unlikely to influence bone or fall risk parameters or exercise or interventions identical in both arms (where WBV was an add-on in one group).

Trials were ineligible if non-RCT, animal studies, population age $<50$ years given by the mean age minus two times the SD or if the participants were younger than 50 years of age, non-English language publications, posters or conference abstracts and if vibration was applied locally, by electrical current, non-standing, with random frequencies, using vibrating insoles or by ultrasound.

\section{Data extraction and quality assessment}

Data were independently extracted by two authors (DBJ, KT), using a standardised data extraction form. For all included studies, information was gathered on country of origin, design, randomisation, population, intervention, adherence, analyses per intention to treat or per protocol and results.

Primary outcomes of interest were fractures and falls, and secondary outcomes were bone parameters including BMD (spine and hip), bone microarchitecture (assessed by high-resolution peripheral quantitative CT (HRpQCT) or bone biopsy), bone turnover markers (carboxy-terminal collagen cross-link (CTX) (bone resorption) or amino terminal propeptide of type I collagen (P1NP) (bone formation)) or calcaneal quantitative ultrasound (BUA).

Data were extracted from the intervention and control groups, and if the WBV was an add-on to exercise, then the exercise and WBV arm was compared with the exercise arm.

The numbers of fractures and the participants contributing with data were extracted in the groups. 


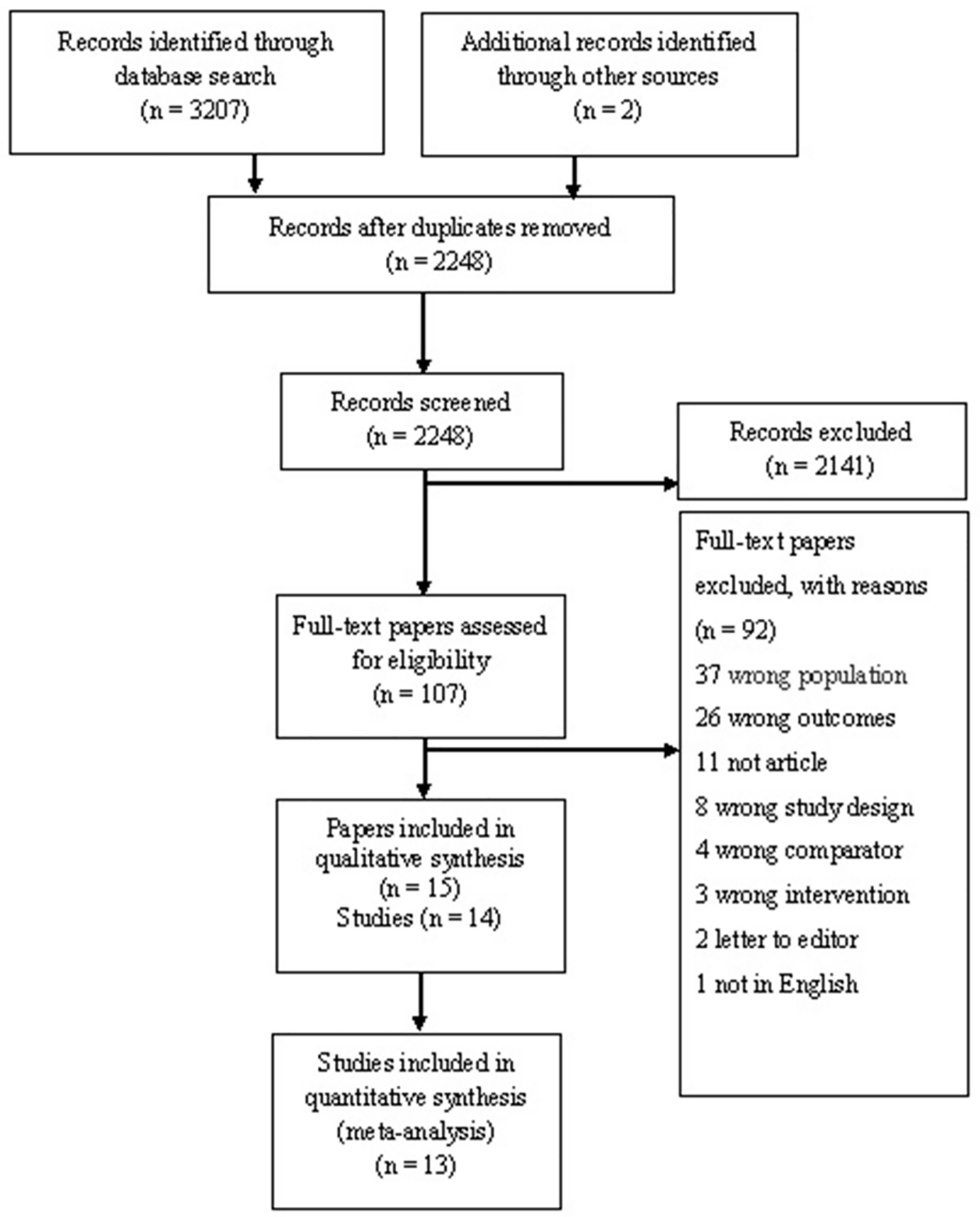

Figure 1 PRISMA (Preferred Reporting Items for Systematic Reviews and Meta-Analyses) flow diagram presenting the literature searches and the included studies.

Regarding falls, the number of falls, the number of participants who experienced falls and the number of participants contributing with data and length of follow-up were extracted. To reduce the clinical heterogeneity, only falls data from the intervention periods of the studies were extracted.

For BMD, bone turnover markers, microarchitecture parameters and BUA, the absolute mean difference (with SDs) from baseline to follow-up were extracted in the intervention and control groups.

If the data were reported different than stated above, the corresponding authors of the included studies were contacted to acquire the data.

The risk of bias for each included study was assessed using the Cochrane 'Risk of bias tool' ${ }^{36}$ The performance biases were divided in patient reported outcomes (falls) and bone property parameters. The quality of evidence was assessed for each outcome using the five GRADE considerations, and summaries of findings were created using the GRADE guidelines. ${ }^{37}$

\section{Strategy for data synthesis and analysis}

The results across studies were pooled by numbers of events calculating relative risk of fractures and for experiencing one or more falls (fallers) with 95\% CI. Fall incident rate ratio per patient year with $95 \%$ CI was calculated using the reported rate of falls (falls per personyear), or the rate of falls in each group were calculated from the total number of falls and the total length of the intervention duration (person-years) for participants contributing with data in each group using STATA V.14. The mean differences in BMD, bone turnover markers, 


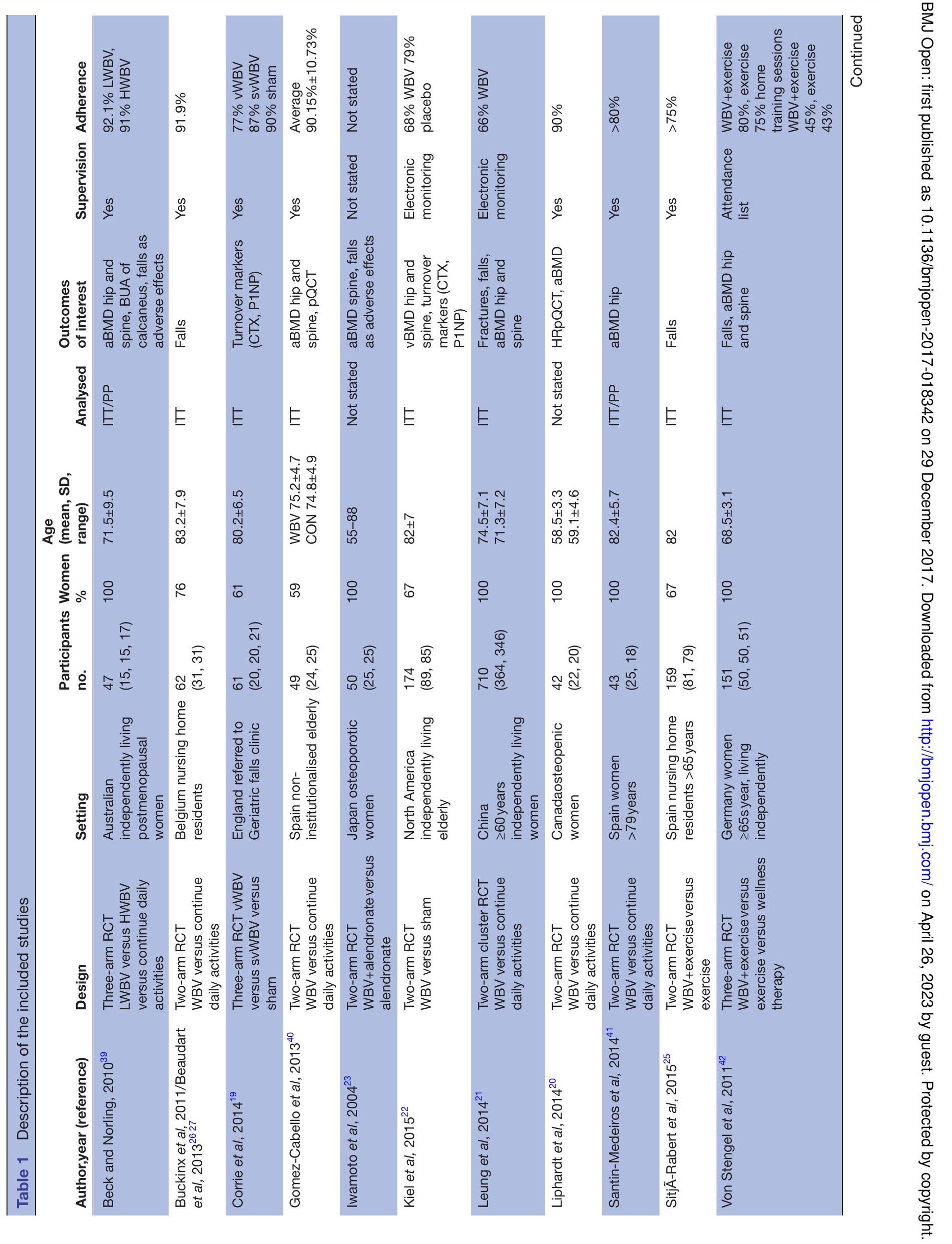


microarchitecture parameters and BUA were pooled calculating the absolute mean difference and 95\% CI. The mean differences were calculated subtracting the baseline means from the follow-up or by multiplying percent change with baseline means. The SDs were calculated using the formula: ((HCI-LCI/2/TINV $(0.05 ; n-1) * \sqrt{ }(n))$, where HCI is the highest value of $95 \%$ CI, LCI the lowest value of $95 \%$ CI and $\mathrm{n}$ the sample size of the group, TINV $(0.05 ; n-1)=$ tvalue for a $95 \%$ CI from a sample size of $n,{ }^{36}$ by using $\mathrm{P}$ values for change over time in Review Manager calculator (RevMan) V.5.3 (Copenhagen: The Nordic Cochrane Centre, The Cochrane Collaboration 2014) or by the formula $\mathrm{SD}=\left(\left(\mathrm{m}_{\mathrm{i}}-\mathrm{m}_{\mathrm{c}}\right) / \mathrm{TINV}(\mathrm{P}\right.$ value; df) $) / \sqrt{ }\left(1 / n_{i}+1 / n_{c}\right)$, where $m_{i}$ is the mean difference in the intervention group, $m_{c}$ is the mean difference in the control group, $\mathrm{df}$ is degrees of freedom, $n_{\mathrm{i}}$ is the sample size in the intervention group and $n_{c}$ is the sample size in the control group. ${ }^{36}$ When cluster randomisation was used, adjustments were applied. ${ }^{36}$ The number of participants contributing with data in each group was used for the calculations if this was reported; otherwise, the number of participants randomised to each group was extracted. Where possible, the longest follow-up times were used (with 2 papers reporting 3-month and 6-month data, 6-month data were used). ${ }^{26}{ }^{27}$ In case of post hoc study extension, the originally planned duration was used. ${ }^{22}$ Calculations were performed using Excel and STATA V.14. To allow for variability among the participants and interventions, the random effect meta-analysis model in RevMan was used. Heterogeneity was assessed by forest plots and the $\mathrm{I}^{2}$ statistics. Preassigned subgroup analyses for sinusoidal vertical and side-alternating WBV were done where possible.

The review protocol was registered 1 April 2016 in Prospective Register of Systematic Reviews (PROSPERO) and reported according to the Preferred Reporting Items for Systematic Reviews and Meta-Analyses (PRISMA) 2009 statement, and the checklist was completed. ${ }^{38}$

\section{RESULTS}

\section{Study selection and characteristics}

A total of 3207 titles and abstracts were initially identified, and after removal of 959 duplicates, 2248 titles and abstracts were screened for relevance. The majority of identified papers were excluded because they described animal studies, were not RCTs or did not meet the definition of the intervention. A total of 107 full-text papers were read and matched to the inclusion and exclusion criteria. Selection of the included studies is illustrated in the PRISMA flow diagram (figure 1). The updated search revealed no new relevant studies.

\section{Study characteristics}

A total of 15 papers (14 studies) met the criteria for the qualitative synthesis and are described in table 1 . The studies were published from 2004 to 2015, with an accumulated population of 1839 (ranging from $42^{20}$ to $710^{21}$ 


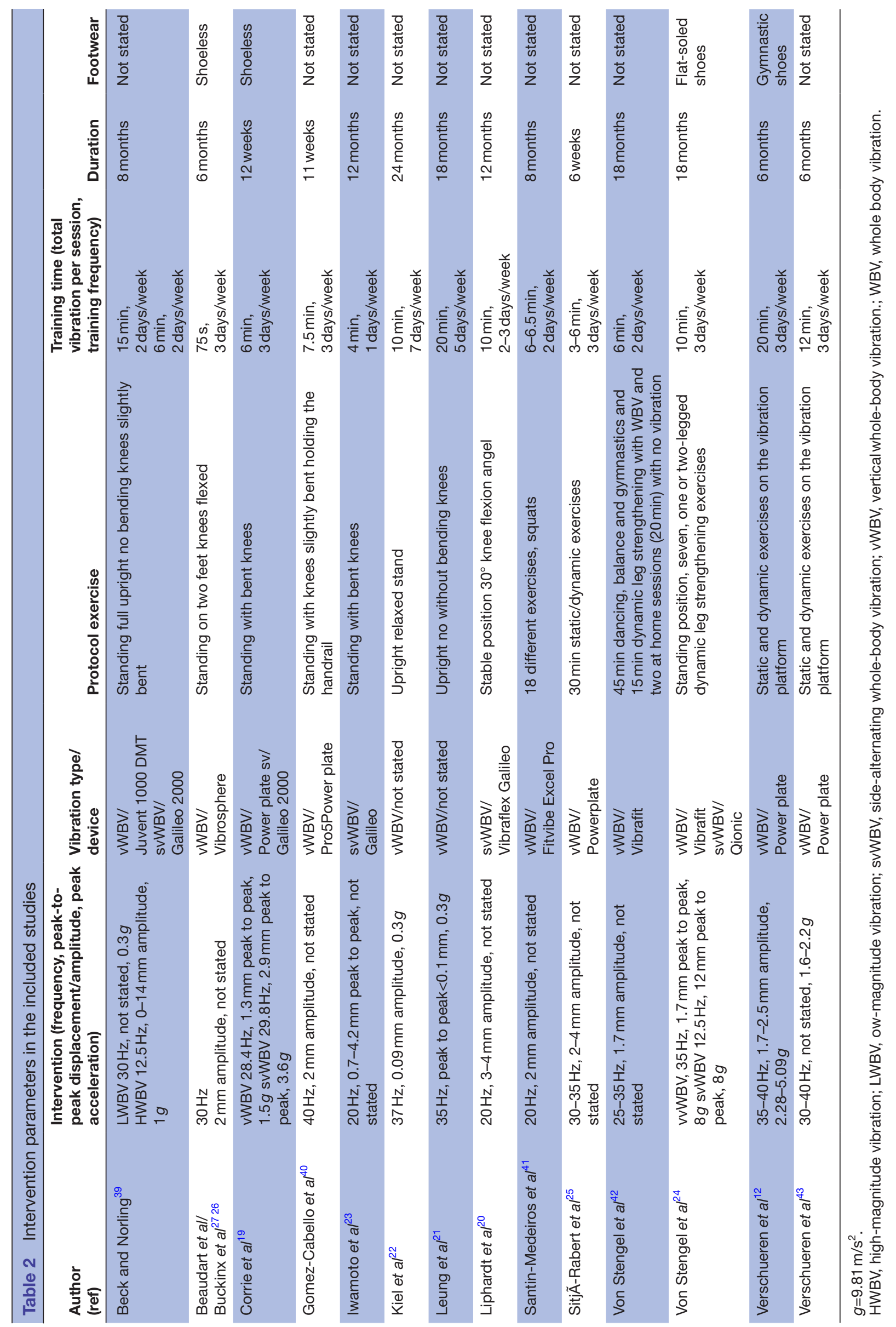




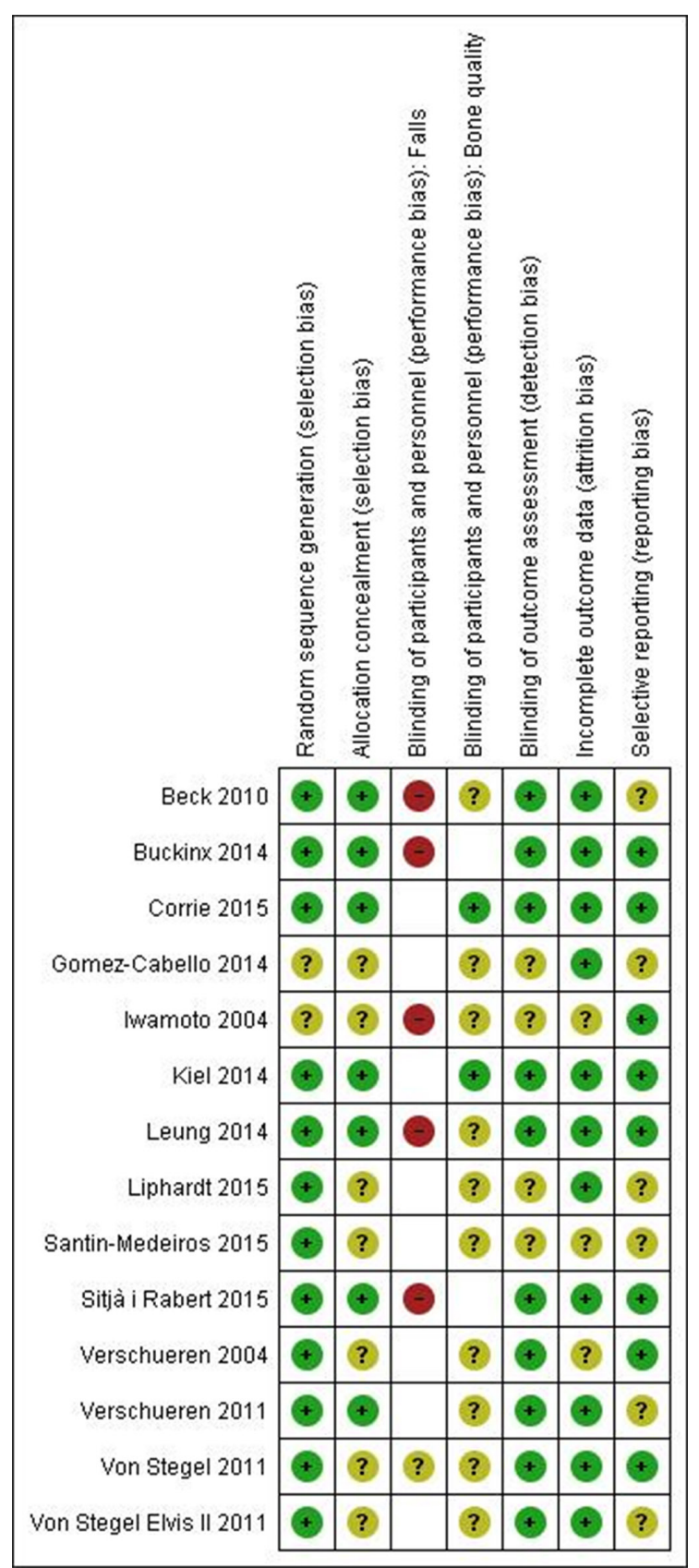

Figure 2 The risk of bias assessment.

participants in the included studies). The mean age of the overall population was 74 years, with $82 \%$ living independently and $90 \%$ being women. All studies were RCT with one trial using cluster randomisation. ${ }^{21}$ The six studies compared WBV with continued daily activities, 202126 39-41 with one study using two different forms of WBV. ${ }^{39}$ Three studies compared WBV with exercise or wellness therapy, 242542 and one study compared WBV with exercise and to continued daily activities. ${ }^{12}$ Two studies compared WBV with sham. ${ }^{19} 22$ One study compared WBV and high or low-dose vitamin D supplementation with no training and high or low-dose vitamin D supplementation. ${ }^{43}$ One study compared WBV and alendronate with no training and alendronate. ${ }^{23}$ Eight trials reported supervised training, $1920252639-4143$ two electronically monitored, ${ }^{21} 22$ two using attendance $\operatorname{logs}{ }^{24} 42$ and two did not state any form of measurement of adherence. ${ }^{12} 23$

The studies varied in the intervention protocols with differences in vibration design, duration and follow-up (table 2). Eleven studies used high-magnitude WBV (HWBV) ( $\geq 1 \mathrm{~g}$ in peak acceleration) $12192023-2640-43$ with two of these studies comparing vertical with side-alternating vibration and wellness therapy/sham vibration. ${ }^{19} 24$ Two studies used low-magnitude WBV (LWBV) $\left(<1 \mathrm{~g}\right.$ in peak acceleration),${ }^{21}{ }^{22}$ and one study compared LWBV to high-magnitude side-alternating $\mathrm{WBV}^{39}$ In the studies, using HWBV, five studies used side-alternating vibration ${ }^{1920232439}$ and nine studies used vertical vibration. $^{12} 19242539-43$ Frequencies ranged from 12.5 to $40 \mathrm{~Hz}$, peak-to-peak displacement ranged from 0.7 to $4.2 \mathrm{~mm}$ and peak acceleration from 0.3 to $8 g$. The exercises were most often vibration spouts lasting from $15 \mathrm{~s}$ to $20 \mathrm{~min}$, from every day to once a week, and the duration of the intervention were from 6 weeks to 24 months. In 2 studies, the participants used flat-soled shoes/gymnastic shoes, ${ }^{12}{ }^{24} 2$ studies described the intervention shoeless, ${ }^{19}{ }^{26}$ while the other 10 studies did not report a protocol for footwear (table 2).

\section{Outcomes}

One study reported fractures as the primary outcome. A total of six studies reported fall data. Three authors were contacted to obtain data on fall rate ${ }^{2325}$ and fall risk, ${ }^{42}$ and this way data were obtained from one trial. ${ }^{25}$

Data on bone parameters were reported in per cent change or preintervention and postintervention measurements in eight studies. The corresponding authors were contacted, ${ }^{12}$ 19-23 394143 and data were obtained this way from three studies. ${ }^{192122}$

In two studies, data were extracted from previous reviews, ${ }^{33}$ which reported to have primary data available from the authors, ${ }^{12} 231$ and in the rest of the studies, the outcomes were calculated as described in the method section.

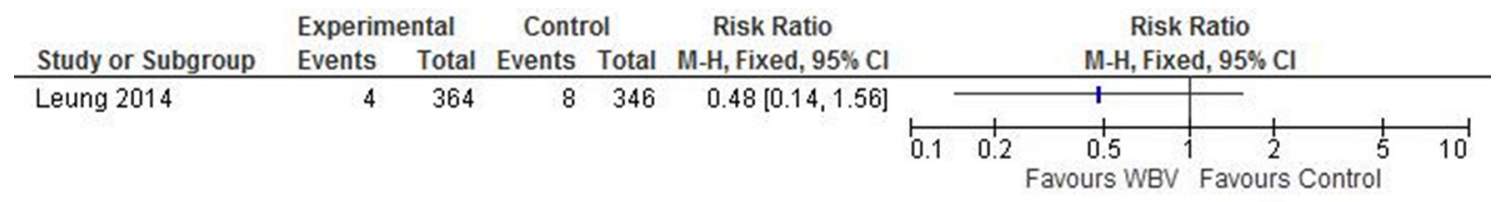

Figure 3 Effect of WBV on the relative risk of experiencing a fracture. WBV, whole-body vibration. 
A

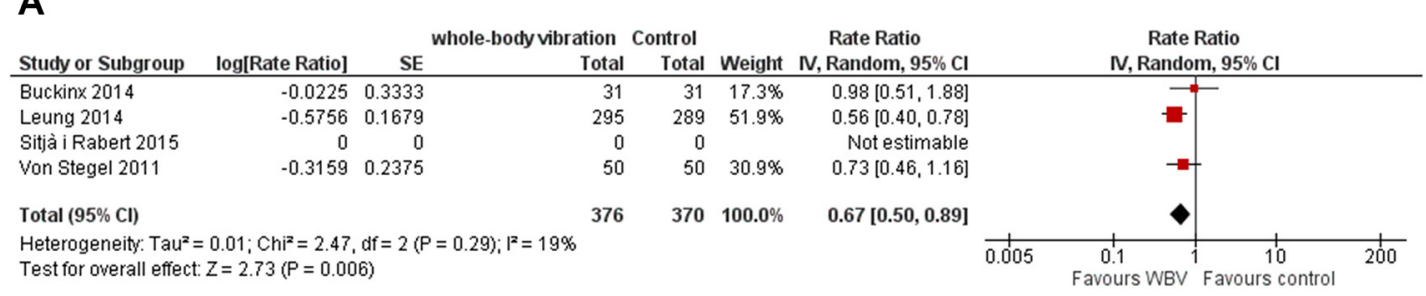

B

\begin{tabular}{|c|c|c|c|c|c|c|c|c|c|c|}
\hline Study or Subgroup & \multicolumn{2}{|c|}{ Whole-body vibration } & \multicolumn{2}{|c|}{ Control } & Weight & $\begin{array}{c}\text { Risk Ratio } \\
\text { M-H, Random, } 95 \% \mathrm{Cl} \\
\end{array}$ & \multicolumn{4}{|c|}{$\begin{array}{c}\text { Risk Ratio } \\
\text { M-H, Random, } 95 \% \mathrm{Cl} \\
\end{array}$} \\
\hline Buckin× 2014 & 9 & 31 & 9 & 31 & $26.0 \%$ & $1.00[0.46,2.18]$ & & & & \\
\hline Leung 2014 & 55 & 295 & 83 & 289 & $71.8 \%$ & $0.65[0.48,0.88]$ & & 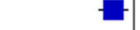 & & \\
\hline Sitjà i Rabert 2015 & 2 & 81 & 0 & 78 & $2.3 \%$ & $4.82[0.23,98.77]$ & & & & \\
\hline Total $(95 \% \mathrm{Cl})$ & & 407 & & 398 & $100.0 \%$ & $0.76[0.48,1.20]$ & & & & \\
\hline Total events & 66 & & 92 & & & & & & & \\
\hline $\begin{array}{l}\text { Heterogeneity: Tau } \\
\text { Test for overall effect }\end{array}$ & $\begin{array}{l}0.05 ; \mathrm{Chi}^{2}= \\
\mathrm{Z}=1.18(\mathrm{P}=\end{array}$ & $=2(\mathrm{~F}$ & $=0.27)$ & $I^{2}=24$ & & & 0.01 & $\begin{array}{l}0.1 \\
\text { Favours WBV }\end{array}$ & $\begin{array}{c}10 \\
\text { Favours control }\end{array}$ & 100 \\
\hline
\end{tabular}

Figure 4 Forest plot of the effect of WBV. (A) The rate ratio of the fall rate/person-years between the WBV and control group. (B) The RR of experiencing one or more falls. Area of each square is proportional to study weight in meta-analysis and horizontal lines represent exact 95\% Cls. Diamonds represent pooled effect estimates from random effects meta-analysis. RR, risk ratio; WBV, whole-body vibration.

\section{Risk of biases within studies}

The majority of studies were categorised as having a low risk of bias in the randomisation with unclear risk of bias in the allocation due to insufficient reporting in half of the studies. The performance bias was categorised as high risk when the participants reported falls and were not blinded to the intervention. One study used wellness therapy in the control group and did not inform the participants of the hypotheses and was thus considered unclear in the risk of performance bias with respect to falls reporting. ${ }^{42}$ Non-blinding of participants were categorised as unclear risk of bias when the outcome were bone parameters. The risk of bias in selective reporting was categorised as low risk if the trial reported all stated outcomes in the papers and was conducted before 2005. After 2005, trials had to be registered online at a registry or having published a study protocol reporting the prespecified outcomes. Figure 2 shows a summary of the risk of bias assessment.

\section{Fractures}

One study reported fractures as a primary outcome (risk ratio (RR) 0.48 (95\% CI 0.14 to 1.56 ), with an intracluster correlation coefficient of 0.000 (figure 3 ).

\section{Falls}

Four studies reported falls as primary outcome. ${ }^{21} 252642$ Three studies reported fallers and the number of falls in total in each group during the intervention, ${ }^{21} 2526$ and one study reported the mean number of falls per participants. ${ }^{42}$ One study reported no events in the control arm in the 6-week intervention and adjusted rate ratio could not be calculated. Pooling the studies with falls reported as outcomes showed a fall rate ratio of 0.67 (95\% CI 0.50 to $0.89, \mathrm{P}=0.006, \mathrm{I}^{2}=19 \%$ ) (figure $4 \mathrm{~A}$ ) in the intervention groups compared with non-intervention and a relative risk of experiencing falls of 0.76 (95\% CI 0.48 to 1.20 , $\mathrm{P}=0.24, \mathrm{I}^{2}=24 \%$ ) (figure 4B).

Two trials reported falls as adverse effects. ${ }^{23}{ }^{39} \mathrm{~A}$ post hoc sensitivity analysis was conducted to assess if the inclusion of these trials would alter the result. In this analysis, a fall rate/person-years rate ratio of 0.65 (95\% CI 0.50 to $0.85, \mathrm{P}=0.002, \mathrm{I}^{2}=8 \%$ ) was found and a relative risk of experiencing falls of 0.67 (95\% CI 0.46 to $0.98, \mathrm{P}=0.04$, $\mathrm{I}^{2}=13 \%$ ) (see online supplementary figure $1 \mathrm{a}, \mathrm{b}$ ).

Post hoc subgroup analyses were conducted to assess the association between the duration and the magnitude of the vibration and falls, duration over 6 months fall rate ratio of $0.61\left(95 \%\right.$ CI 0.47 to $0.80, \mathrm{P}=0.0004, \mathrm{I}^{2}=0 \%$, two studies), duration over 6 months and relative risk of experiencing falls of 0.65 ( $95 \%$ CI 0.48 to $0.88, \mathrm{P}=0.005, \mathrm{I}^{2}=0 \%$, one study), low-magnitude vibration fall rate ratio of 0.56 ( $95 \%$ CI 0.40 to $0.78, \mathrm{P}=0.0006$, one study), high-magnitude vibration fall rate ratio of 0.80 (95\% CI 0.55 to 1.18 , $\mathrm{P}=0.26, \mathrm{I}^{2}=0 \%$, two studies) (see online supplementary figure $2 \mathrm{a}-\mathrm{c})$.

\section{Bone mineral density}

Seven studies reported data on lumbar spine BMD. $^{12} 212324394042$ The results showed no overall effect with a mean difference of $0.00(95 \%$ CI -0.00 to -0.01 , $\mathrm{P}=0.11, \mathrm{I}^{2}=22 \%$ ) (figure $5 \mathrm{~A}$ ). Six studies reported data on total hip BMD, ${ }^{12} 2140-43$ showing similar results with a mean difference of 0.00 (95\% CI -0.00 to $0.01, \mathrm{P}=0.27$, $\mathrm{I}^{2}=50 \%$ ) (figure $5 \mathrm{~B}$ ). Subgroup analyses with vertical and side-alternation vibration explained $44.5 \%$ of the heterogeneity in the lumbar spine BMD, and side-alternation 
A

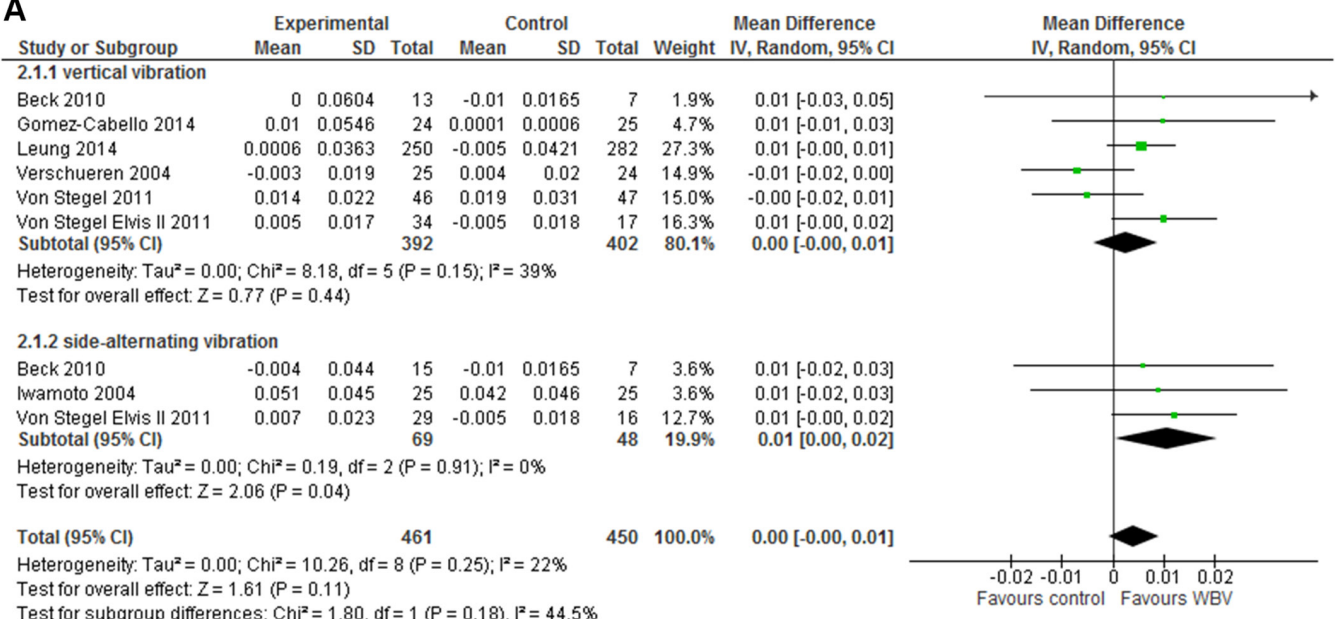

B

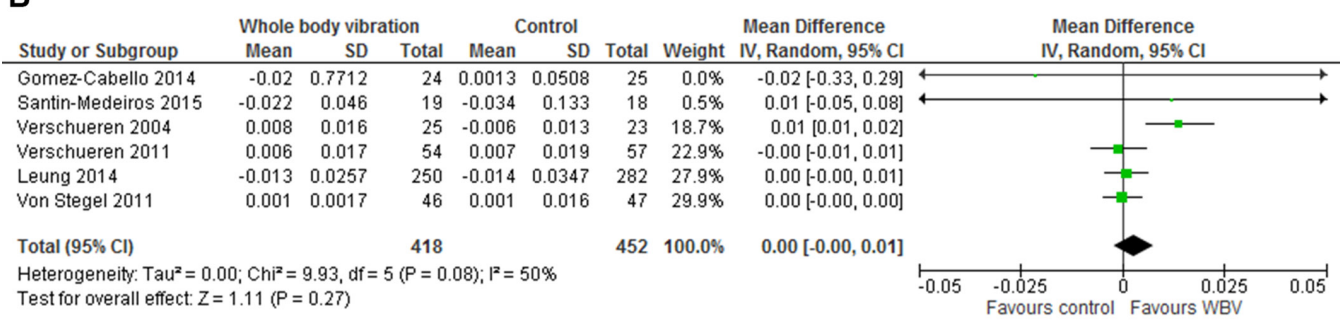
$\begin{array}{llll}\text { C } & \text { Experimental } & \text { Control } & \text { Mean Difference } \\ \text { Study or Subgroup } & \text { Mean } & \text { SD Total Mean } & \text { SD Total Weight IV, Random, } 95 \% \mathrm{Cl}\end{array}$ Study or Subgroup Mean SD Total Mean SD Total Weight IV, Random, $95 \% \mathrm{Cl} \quad$ Mean Difference

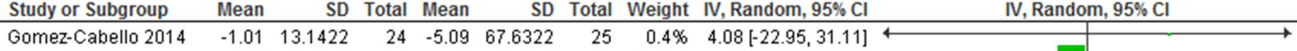
$\begin{array}{lllllllll}\text { Liphardt } 2015 & -2.7 & 2.7727 & 17 & -2 & 1.7729 & 14 & 99.6 \% & -0.70[-2.31,0.91]\end{array}$

Total $(95 \% \mathrm{Cl}) \quad 41 \quad 39 \quad 100.0 \% \quad-0.68[-2.29,0.93]$ Heterogeneity: $\operatorname{Tau}^{2}=0.00 ; \mathrm{Chi}^{2}=0.12, \mathrm{df}=1(\mathrm{P}=0.73) ; \mathrm{I}^{2}=0 \%$ Test for overall effect: $Z=0.83(P=0.41)$

D

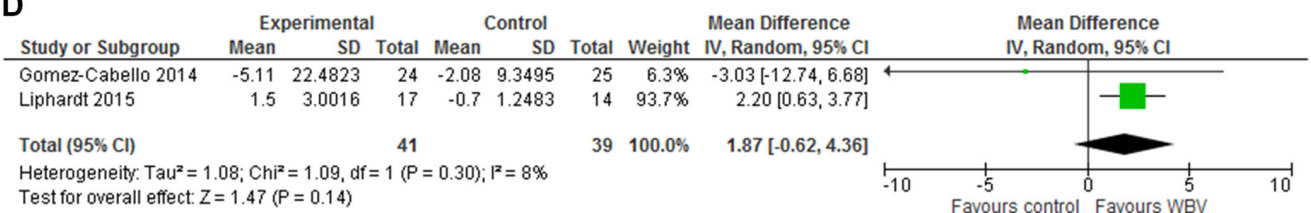

Figure 5 The effect of WBV exercise in forest plots on (A) areal BMD of the lumbar spine with weighted mean difference and $95 \% \mathrm{Cl}$, divided in subgroups with vertical vibration and side-alternating vibration, (B) areal BMD in total hip with weighted mean difference and $95 \% \mathrm{Cl},(\mathrm{C})$ volumetric BMD of the distal tibia with weighted mean difference with $95 \% \mathrm{Cl}$ and (D) WBVs effect on volumetric BMD of the distal radius with weighted mean difference and $95 \% \mathrm{Cl}$. Area of each square is proportional to study weight in meta-analysis and horizontal lines represent exact $95 \% \mathrm{Cl}$. Diamonds represent pooled effect estimates from random effects meta-analysis. BMD, bone mineral density; WBV, whole-body vibration.

vibration showed a mean difference of 0.01 (95\% CI 0.00 to $0.02, \mathrm{P}=0.04, \mathrm{I}^{2}=0 \%$ ) with 117 participants. All studies reporting $\mathrm{BMD}$ in total hip used vertical vibration.

One study reported change in total proximal femoral trabecular BMD and change in integral lumbar spine vertebral BMD. ${ }^{22}$ The results from the originally planned duration of 24 months showed no effect on integral lumbar spine vertebral BMD with a mean difference of $0.00(95 \%$ CI -0.00 to 0.00$)$ and total femoral trabecular BMD mean difference of $0.00(95 \%$ CI -0.00 to 0.01$)$ (see online supplementary figure $3 \mathrm{a}, \mathrm{b})$. Two studies reported volumetric BMD (vBMD) of radius and tibia using HRpQCT ${ }^{20}$ or quantitative CT (pQCT) scans. ${ }^{37}$ The results for the ultradistal site using HRpQCT and a $4 \%$ site in tibia and radius using pQCT were combined in forest plots showing no statistically significant effects with a vBMD tibia mean difference of -0.68 (95\% CI -2.29 to $\left.0.93, \mathrm{P}=0.41, \mathrm{I}^{2}=0\right)$ and a vBMD radius mean difference of $1.87\left(95 \% \mathrm{CI}-0.62\right.$ to $\left.4.36, \mathrm{P}=0.30, \mathrm{I}^{2}=8 \%\right)$ (figure 5C,D).

\section{Bone microarchitecture}

One study reported measurements of cortical porosity (Ct.Po) and trabecular BMD (tbBMD) ${ }^{20}$ using HRpQCT. We refrained from performing a meta-analysis due to the limited data (see online supplementary figure 4). In tibia, WBV compared with control showed an increase in mean difference in Ct.Po of $0.20 \%$ (95\% CI -0.25 to 0.65$)$ and decrease in tbBMD mean difference $-0.3 \mathrm{mg} \mathrm{HA} / \mathrm{cm}^{3}$ $(95 \% \mathrm{CI}-0.58$ to 0.02$)$. In radius, WBV compared with 
A

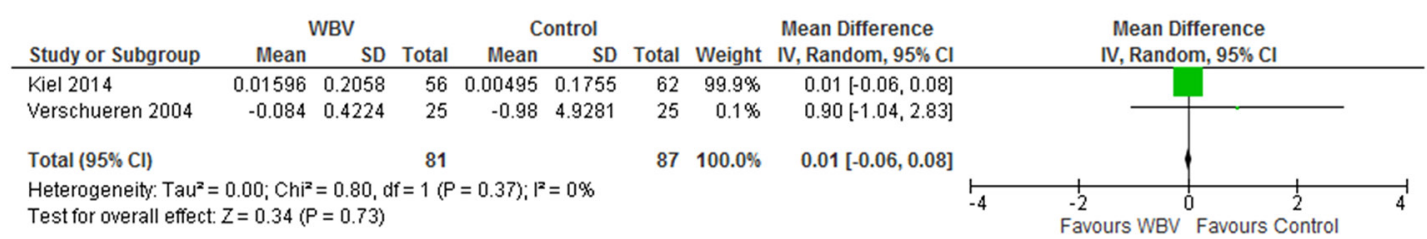

B

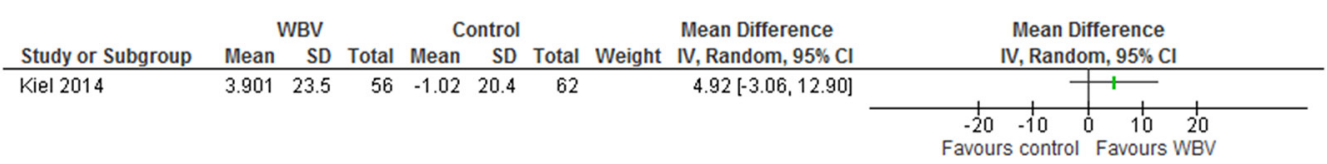

Figure 6 Presents the effect of WBV exercise on bone resorption markers in forest plot with CTX and the reported effect on bone formation marker amino terminal P1NP. Area of each square is proportional to study weight in meta-analysis and horizontal lines represent exact $95 \%$ Cls. Diamonds represent pooled effect estimates from random effects metaanalysis. CTX, carboxy-terminal collagen cross-link; P1NP, propeptide of type I collagen; WBV, whole-body vibration.

no intervention showed an increase mean difference in Ct.Po of $0.10 \%$ (95\% CI -0.15 to 0.35$)$ and decrease in tbBMD mean difference $-0.90 \mathrm{mg} \mathrm{HA} / \mathrm{cm}^{3}(95 \% \mathrm{CI}$ -0.90 to 2.10 ) (see online supplementary figure 4 ).

\section{Bone turnover markers}

One study reported data on the bone resorption marker CTX $^{12}$ and two studies on both CTX and the bone formation marker P1NP. ${ }^{192}$ One of the studies reported log transformed CTX and $\mathrm{P}_{1} \mathrm{NP}^{19}$ and no untransformed data could be obtained from the authors. The result for the meta-analysis on CTX was a mean difference of $0.01 \mathrm{ng} / \mathrm{mL}\left(95 \% \mathrm{CI}-0.06\right.$ to $\left.0.08, \mathrm{P}=0.73, \mathrm{I}^{2}=0\right)$ and with data available from only one trial, the result for P1NP was a mean difference of $4.92 \mathrm{ng} / \mathrm{mL}$ (95\% CI -3.06 to 12.90$)$ (figure 6A,B).

\section{Calcaneal BUA}

A single study reported calcaneal BUA mean change in comparing two vibration groups with a control group, ${ }^{39}$ we refrained from performing a meta-analysis due to the limited data (see online supplementary figure 5). The low magnitude vertical vibration group had a mean difference of $1.99 \mathrm{~dB} / \mathrm{MHz}$ (95\% CI -0.84 to 4.82 ) and the high-magnitude side-altering vibration group a mean change of $4.69 \mathrm{~dB} / \mathrm{MHz}$ (95\% CI 1.61 to 7.77 ) compared with the controls (see online supplementary figure 5 ).

\section{Quality assessment}

Quality of evidence was assessed for each outcome (table 3). For the outcome of fractures, the evidence was downgraded for imprecision due to the $95 \% \mathrm{CI}$ around the pooled estimate of effect includes both the possibility of no effect and appreciable benefit. The evidence for falls rate was downgraded for study limitations due to non-blinding of the participants. The risk of falls was downgraded for imprecision and study limitations due to non-blinding of the participants. Bone parameters were all downgraded for indirectness since they are surrogate markers for bone strength. Regarding bone parameters, the outcomes were downgraded for imprecision if the 95\% CI around the pooled estimate of effect includes both the possibility of no effect and appreciable benefit and for inconsistency if the $\mathrm{I}^{2}$ statistics showed substantial heterogeneity. Publication bias could not be assessed by a funnel plot with Egger's test since all of the meta-analyses contained less than 10 studies. $^{36}$

\section{DISCUSSION}

This systematic review and meta-analysis provides evidence that WBV exercise reduces fall rate in adults above 50 years of age. We found a tendency in reduction of the proportion of fallers, no overall effect on BMD, whereas only sparse data were available regarding bone microarchitecture parameters, bone turnover markers and BUA. One study reported fractures showing non-significant fracture reduction.

\section{Strengths and limitations}

This study had some limitations. By not including nonEnglish language literature and not extracting data from grey literature or adverse effects, the risk of selection bias exists. Looking at the studies reporting falls as adverse effects in the included studies, the WBV reduces the falls rate and risk in agreement with our findings.

Only one study had fractures as primary outcome and had a low fracture rate. ${ }^{21}$ The studies contributing with falls data were non-blinded which could be important when reporting falls. However, all studies included in the primary falls analysis did record falls prospectively limiting the risk of recall bias. ${ }^{21} 252642$ The populations in the studies consisted of $82 \%$ community-dwelling adults 
Table 3 Summary of findings table presents the findings and the quality of each outcome using the GRADE considerations WBV compared with usual care for fracture risk Bibliography

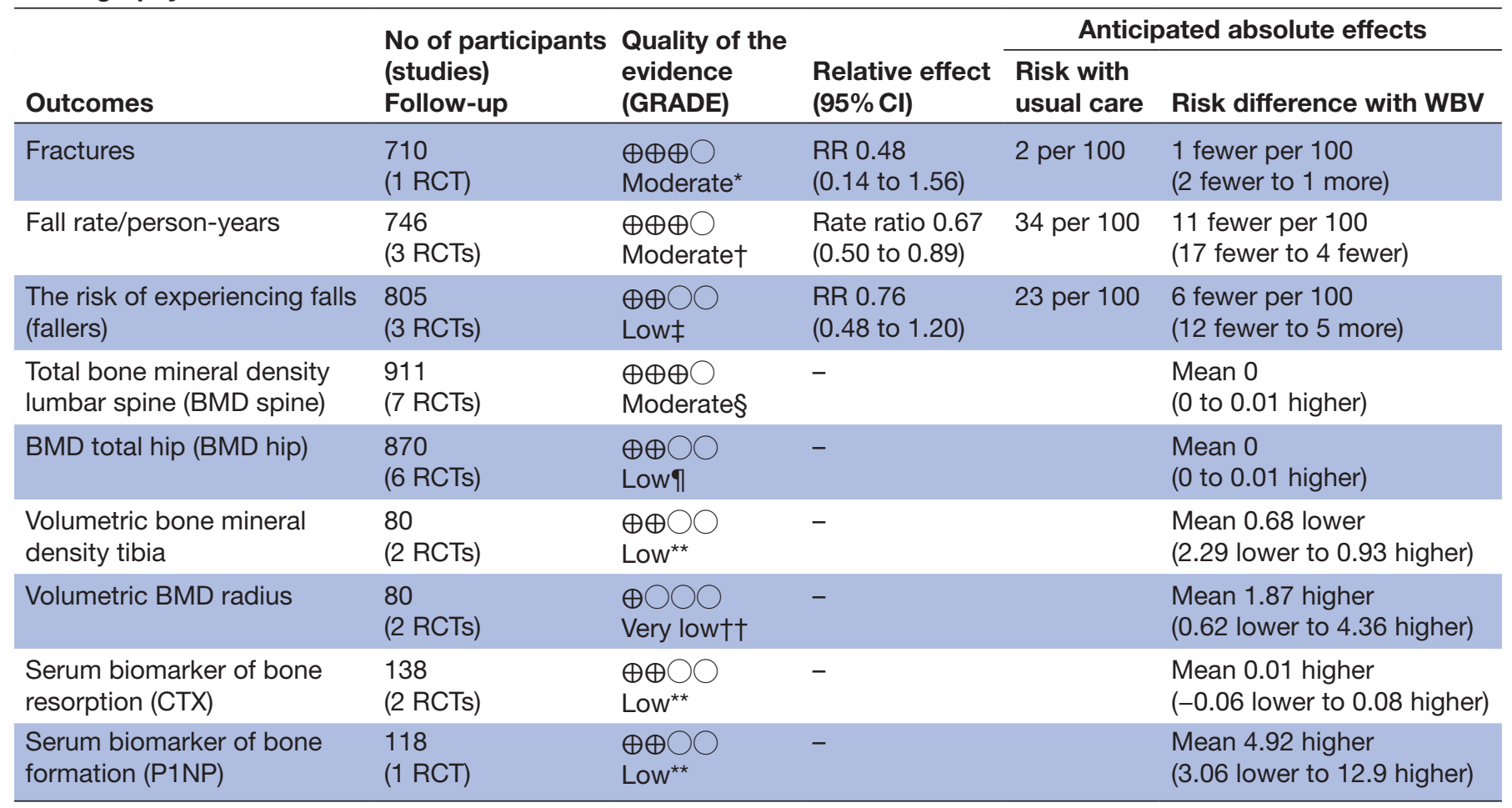

The risk in the intervention group (and its $95 \% \mathrm{Cl}$ ) is based on the assumed risk in the comparison group and the relative effect of the intervention (and its $95 \% \mathrm{Cl}$ ).

High quality: We are very confident that the true effect lies close to that of the estimate of the effect; Moderate quality: We are moderately confident in the effect estimate: The true effect is likely to be close to the estimate of the effect, but there is a possibility that it is substantially different; Low quality: Our confidence in the effect estimate is limited: The true effect may be substantially different from the estimate of the effect; Very low quality: We have very little confidence in the effect estimate: The true effect is likely to be substantially different from the estimate of effect.

*Serious imprecision, due to the $95 \% \mathrm{Cl}$ around the estimate of effect includes both the possibility of no effect and appreciable benefit. †Serious study limitations - lack of blinding of the participants reporting falls.

¥Serious study limitations - lack of blinding of the participants reporting fall, and serious imprecision, due to the $95 \% \mathrm{Cl}$ around the pooled estimate of effect includes both the possibility of no effect and appreciable benefit.

§Indirectness (surrogate marker for bone strength).

IIndirectness, and statistical heterogeneity

${ }^{*}$ Indirectness, and imprecision due to the $95 \% \mathrm{Cl}$ around the estimate of effect includes both the possibility of no effect and appreciable benefit.

††indirectness, and imprecision due to the $95 \% \mathrm{Cl}$ around the estimate of effect includes both the possibility of no effect and appreciable benefit and statistical heterogeneity.

BMD, bone mineral density; CTX, carboxy-terminal collagen cross-link; GRADE, Working Group grades of evidence; P1NP, propeptide of type I collagen; RCTs, randomised controlled trials; RR, risk ratio; WBV, whole-body vibration.

with $90 \%$ being female, making the results generalisable only to people with similar characteristics.

Strengths of this review include that the evidence is obtained from RCTs, followed the PRISMA guidelines of reporting and was registered at PROSPERO to improve transparency. A thorough literature search was conducted with assistance from a research librarian and we furthermore performed a hand search of the reference lists of included papers and earlier reviews references. ${ }^{28} 29$ 32-35 The risk of selection bias was reduced by having two independent reviewers select the papers and extract the data. In the systematic review, all outcomes were assessed regarding quality using the GRADE guidelines where fracture is classified as a critical outcome. ${ }^{37}$ We classified falls as an important outcome and bone parameters being of limited importance as surrogate makers for fracture risk. ${ }^{37}$ We only pooled homogeneous outcomes in the meta-analysis leading to low statistical heterogeneity in the falls analysis with moderate statistical heterogeneity regarding BMD of the hip and spine. Preassigned subgroup analysis for vertical versus side-alternating vibration could explain $44.5 \%$ of the heterogeneity in the lumbar spine analysis, whereas regarding total hip $B M D$, all studies used vertical vibration and no subgroup 
analysis was performed. Meta-regression analysis was not performed due to the insufficient number of studies in the analysis. ${ }^{36}$

\section{Comparisons with other studies and reviews}

Prior reviews of exercise have shown that exercise programme designed to prevent falls in older adults also seem to prevent injuries caused by falls, including fractures. ${ }^{445}$ The majority of these exercise programme included balance training, functional training and strengthening exercises. Earlier reviews have shown that WBV have balance improving capabilities and the ability to improve muscle strength of the lower extremities, ${ }^{27-31}$ and WBV might thus prevent fractures by its fall-reducing capacity or by lowering the impact of a fall.

Our meta-analysis shows that rate of falls can be reduced and suggests a reduction in the proportion of fallers. The number needed to treat to prevent one fall was 11 (table 3). Sustaining a fall increases the risk of injury and reducing the number of times an individual falls, even if not the number of fallers may have clinical and economic relevance to the individual and to society. Falls are very prevalent among the ageing population with one in every three adults aged $65+$ years old experiencing a fall every year. ${ }^{6}$ Due to an ageing population, a focus on interventions capable of reducing falls seems of utmost importance. ${ }^{10}$ Prior systematic reviews have shown that other exercise programmes can reduce fall rate through muscle strength and balance training, and it has been found that exercising for a period of more than 3 hours per week is associated with a larger decrease in fall rate. ${ }^{46} \mathrm{WBV}$ exercise consists of shorter workouts and with the ability to stand as the only requirement for physical function. With the available data the analysis shows a fall reduction in the vibration groups with low heterogeneity, and with the observational power of the post hoc subgroup analyses, we found an association between studies with duration longer than 6 months and a larger reduction in falls.

To our knowledge, this is the first meta-analysis conducted on WBV and falls but earlier findings of a positive effect on surrogate markers for falls (balance and muscle strength) ${ }^{27-31}$ can be viewed as an improvement in important risk factors for falls in agreement with our findings.

Our results on BMD are consistent with other systematic reviews, showing no overall effect on BMD. ${ }^{31-34}$ Earlier reviews suggested a positive effect on BMD in adolescents ${ }^{32}$ and in a subgroup analysis with improvements after LWBV on lumbar spine BMD $^{33}$ and HWBV on total hip BMD. ${ }^{32}$ We found a similar but small effect of side alternating vibration on lumbar spine BMD. In contrast to others, this systematic review also comprehensively assessed other bone parameters, that is, bone microarchitecture, turnover markers and BUA. We found one study assessing cortical porosity and trabecular BMD of tibia and radius ${ }^{20}$ with no overall effect, which is in line with results found in a younger age group. ${ }^{47}$ We found no effect on bone resorption markers in line with studies in younger participants. ${ }^{48} 49$ One study in this review had a positive effect in bone formation markers, but with logarithmic transformed data it could not be pooled with non-transformed data $^{19}$ (see online supplementary figure 6). One study looked at BUA of the calcaneus showing a positive effect $^{39}$ in conflict with earlier findings from younger participants. ${ }^{47}$ Animal data suggest an effect of WBV on bone strength, ${ }^{15-18}$ but the same effect in humans is not evident. Reasons for this include diversities in training protocols, duration, adherence, damping of the vibration by the use of shoes and different standing positions on the vibration plates.

In summary, the the evidence from this systematic review indicate that WBV may reduce fall rate with moderate certainty and the risk of falls with low certainty. Future trials could enhance the certainty by systematically reporting falls when monitoring adverse effects, and if possible by blinding participants. The quality of evidence for the effect on bone parameters is moderate to low, partly since they are surrogate markers of fracture risk, and future research should focus on the critical outcome fractures with larger trial sizes and adequate follow-up.

\section{CONCLUSION}

In conclusion, our data show a reduced rate of falls by WBV. Only one study reported fractures showing a non-significant reduction. We found no effect on BMD, and the data on microarchitecture and bone turnover markers were sparse. WBV exercise could be implemented in current falls prevention guidelines. It might potentially reduce fractures by reducing falls, but the impact on fractures needs further larger adequately powered studies.

Acknowledgements We would like to thank associate professor Carsten Bogh Juhl for extensive help and support in performing the statistical analyses and research librarian PhD Mette Brandt Eriksen for the help with the literature searches. We would also like to acknowledge Professor Dr Douglas P Kiel, PhD Mercè Sitjà-Rabert and Dr Katherine Brooke-Wavell for sending us the data from their studies and the grant from the National Institute on Aging (R01 AG025489) which generated the data from the VIBES trial.

Contributors All authors helped in the conception and design of the work and interpretation of data. Three authors reviewed the papers (DBJ, KT and JR) and two authors extracted the data (DBJ, KT). DBJ, TM and JR did the first draft and all authors revised it critically for important intellectual content. All authors approved the final version for publication. DBJ is guarantor.

Funding This study was supported by funding from the Odense University Hospital, The Region of Southern Denmark PhD foundation, the Faculty of Health Sciences, University of Southern Denmark.

Competing interests None declared.

Provenance and peer review Not commissioned; externally peer reviewed.

Data sharing statement No additional data are available.

Open Access This is an Open Access article distributed in accordance with the Creative Commons Attribution Non Commercial (CC BY-NC 4.0) license, which permits others to distribute, remix, adapt, build upon this work non-commercially, and license their derivative works on different terms, provided the original work is properly cited and the use is non-commercial. See: http://creativecommons.org/ licenses/by-nc/4.0/ 
(c) Article author(s) (or their employer(s) unless otherwise stated in the text of the article) 2017. All rights reserved. No commercial use is permitted unless otherwise expressly granted.

\section{REFERENCES}

1. Hernlund $E$, Svedbom A, Ivergård M, et al. Osteoporosis in the European Union: medical management, epidemiology and economic burden. A report prepared in collaboration with the International Osteoporosis Foundation (IOF) and the European Federation of Pharmaceutical Industry Associations (EFPIA). Arch Osteoporos 2013;8:136.

2. Johnell O, Kanis JA. An estimate of the worldwide prevalence and disability associated with osteoporotic fractures. Osteoporos Int 2006;17:1726-33.

3. Kanis JA, Johnell O. Requirements for DXA for the management of osteoporosis in Europe. Osteoporosis International 2005;16:229-38.

4. Tinetti ME, Doucette J, Claus E, et al. Risk factors for serious injury during falls by older persons in the community. J Am Geriatr Soc 1995;43:1214-21.

5. Cummings SR, Nevitt MC. Non-skeletal determinants of fractures: the potential importance of the mechanics of falls. Study of Osteoporotic Fractures Research Group. Osteoporos Int 1994;4:67-70.

6. Blake AJ, Morgan K, Bendall MJ, et al. Falls by elderly people at home: prevalence and associated factors. Age Ageing 1988; 17:365-72.

7. Boutroy S, Khosla S, Sornay-Rendu E, et al. Microarchitecture and peripheral BMD are impaired in postmenopausal white women with fracture independently of total hip T-score: an international multicenter study. J Bone Miner Res 2016;31:1158-66.

8. Vasikaran $S$, Eastell R, Bruyère $O$, et al. Markers of bone turnover for the prediction of fracture risk and monitoring of osteoporosis treatment: a need for international reference standards. Osteoporosis International 2011;22:391-420.

9. Curtis EM, van der Velde R, Moon RJ, et al. Epidemiology of fractures in the United Kingdom 1988-2012: Variation with age, sex, geography, ethnicity and socioeconomic status. Bone 2016:87:19-26.

10. Blain H, Masud T, Dargent-Molina P, et al. A Comprehensive Fracture Prevention Strategy in Older Adults: The European Union Geriatric Medicine Society (EUGMS) Statement. J Nutr Health Aging 2016;20:647-52.

11. Bogaerts A, Delecluse C, Claessens AL, et al. Impact of whole-body vibration training versus fitness training on muscle strength and muscle mass in older men: a 1-year randomized controlled trial. J Gerontol A Biol Sci Med Sci 2007;62:630-5.

12. Verschueren SM, Roelants M, Delecluse C, et al. Effect of 6-month whole body vibration training on hip density, muscle strength, and postural control in postmenopausal women: a randomized controlled pilot study. J Bone Miner Res 2004;19:352-9.

13. Gusi N, Raimundo A, Leal A. Low-frequency vibratory exercise reduces the risk of bone fracture more than walking: a randomized controlled trial. BMC Musculoskelet Disord 2006;7:92.

14. Cheung WH, Mok HW, Qin L, et al. High-frequency whole-body vibration improves balancing ability in elderly women. Arch Phys Med Rehabil 2007;88:852-7.

15. Rubin C, Turner AS, Bain S, et al. Anabolism. Low mechanical signals strengthen long bones. Nature 2001;412:603-4.

16. Rubin C, Turner AS, Mallinckrodt C, et al. Mechanical strain, induced noninvasively in the high-frequency domain, is anabolic to cancellous bone, but not cortical bone. Bone 2002;30:445-52.

17. Pichler $\mathrm{K}$, Loreto $\mathrm{C}$, Leonardi $\mathrm{R}$, et al. RANKL is downregulated in bone cells by physical activity (treadmill and vibration stimulation training) in rat with glucocorticoid-induced osteoporosis. Histol Histopathol 2013;28:1185-96.

18. Pollock RD, Woledge RC, Mills KR, et al. Muscle activity and acceleration during whole body vibration: effect of frequency and amplitude. Clin Biomech 2010;25:840-6.

19. Corrie H, Brooke-Wavell K, Mansfield NJ, et al. Effects of vertical and side-alternating vibration training on fall risk factors and bone turnover in older people at risk of falls. Age Ageing 2015;44:115-22.

20. Liphardt AM, Schipilow J, Hanley DA, et al. Bone quality in osteopenic postmenopausal women is not improved after 12 months of whole-body vibration training. Osteoporos Int, 2015;2620:911.

21. Leung KS, Li CY, Tse YK, et al. Effects of 18-month low-magnitude high-frequency vibration on fall rate and fracture risks in 710 community elderly--a cluster-randomized controlled trial. Osteoporos Int 2014;25:1785-95.

22. Kiel DP, Hannan MT, Barton BA, et al. Low-magnitude mechanical stimulation to improve bone density in persons of advanced age: a randomized, placebo-controlled trial. J Bone Miner Res 2015;30:1319-28.

23. Iwamoto J, Takeda T, Sato $\mathrm{Y}$, et al. Effect of whole-body vibration exercise on lumbar bone mineral density, bone turnover, and chronic back pain in post-menopausal osteoporotic women treated with alendronate. Aging Clin Exp Res 2005;17:157-63.

24. Von Stengel S, Kemmler W, Bebenek M, et al. Effects of whole-body vibration training on different devices on bone mineral density. Med Sci Sports Exerc 2011;43:1071-9.

25. Sitjà-Rabert M, Martínez-Zapata MJ, Fort Vanmeerhaeghe $A$, et al. Effects of a whole body vibration (WBV) exercise intervention for institutionalized older people: a randomized, multicentre, parallel, clinical trial. J Am Med Dir Assoc 2015;16:125-31.

26. Buckinx F, Beaudart C, Maquet D, et al. Evaluation of the impact of 6-month training by whole body vibration on the risk of falls among nursing home residents, observed over a 12-month period: a single blind, randomized controlled trial. Aging Clin Exp Res 2014;26:369-76.

27. Beaudart C, Maquet D, Mannarino M, et al. Effects of 3 months of short sessions of controlled whole body vibrations on the risk of falls among nursing home residents. BMC Geriatr 2013;13:42.

28. Lam FM, Lau RW, Chung RC, et al. The effect of whole body vibration on balance, mobility and falls in older adults: a systematic review and meta-analysis. Maturitas 2012;72:206-13.

29. Sitjà-Rabert M, Rigau D, Fort Vanmeerghaeghe $A$, et al. Efficacy of whole body vibration exercise in older people: a systematic review. Disabil Rehabil 2012;34:883-93.

30. Orr R. The effect of whole body vibration exposure on balance and functional mobility in older adults: a systematic review and metaanalysis. Maturitas 2015;80:342-58.

31. Rogan S, de Bruin ED, Radlinger L, et al. Effects of whole-body vibration on proxies of muscle strength in old adults: a systematic review and meta-analysis on the role of physical capacity level. Eur Rev Aging Phys Act 2015;12:12:12.

32. Lau RW, Liao LR, Yu F, et al. The effects of whole body vibration therapy on bone mineral density and leg muscle strength in older adults: a systematic review and meta-analysis. Clin Rehabil 2011;25:975-88.

33. Slatkovska L, Alibhai SM, Beyene J, et al. Effect of whole-body vibration on BMD: a systematic review and meta-analysis. Osteoporos Int 2010;21:1969-80.

34. Oliveira LC, Oliveira RG, Pires-Oliveira DA. Effects of whole body vibration on bone mineral density in postmenopausal women: a systematic review and meta-analysis. Osteoporos Int 2016;27:2913-33.

35. Ma C, Liu A, Sun M, et al. Effect of whole-body vibration on reduction of bone loss and fall prevention in postmenopausal women: a meta-analysis and systematic review. J Orthop Surg Res 2016;11:24.

36. HigginsJPT GSe. Cochrane Handbook for Systematic Reviews of Interventions Version 5.1.0. (accessed Mar 2011).

37. Schünemann HBJ, Guyatt G. Oxman A GRADE handbook for grading quality of evidence and strength of recommendations. The GRADE Working Group. (accessed Oct 2013).

38. Moher D, Liberati A, Tetzlaff J, et al. Preferred reporting items for systematic reviews and meta-analyses: the PRISMA statement. $J$ Clin Epidemiol 2009;62:1006-12.

39. Beck BR, Norling TL. The effect of 8 mos of twice-weekly low- or higher intensity whole body vibration on risk factors for postmenopausal hip fracture. Am J Phys Med Rehabil 2010;89:997-1009.

40. Gómez-Cabello A, González-Agüero A, Morales S, et al. Effects of a short-term whole body vibration intervention on bone mass and structure in elderly people. J Sci Med Sport 2014;17:160-4.

41. Santin-Medeiros F, Santos-Lozano A, Rey-López JP, et al. Effects of eight months of whole body vibration training on hip bone mass in older women. Nutr Hosp 2015;31:1654-9.

42. von Stengel S, Kemmler W, Engelke K, et al. Effects of whole body vibration on bone mineral density and falls: results of the randomized controlled ELVIS study with postmenopausal women. Osteoporosis International 2011;22:317-25.

43. Verschueren SM, Bogaerts A, Delecluse C, et al. The effects of whole-body vibration training and vitamin $D$ supplementation on muscle strength, muscle mass, and bone density in institutionalized elderly women: a 6-month randomized, controlled trial. J Bone Miner Res 2011;26:42-9. 
44. El-Khoury F, Cassou B, Charles MA, et al. The effect of fall prevention exercise programmes on fall induced injuries in community dwelling older adults: systematic review and meta-analysis of randomised controlled trials. BMJ 2013;347:f6234.

45. Gillespie LD, Robertson MC, Gillespie WJ, et al. Interventions for preventing falls in older people living in the community. Cochrane Database Syst Rev 2012;9:Cd007146.

46. Sherrington $\mathrm{C}$, Michaleff ZA, Fairhall N, et al. Exercise to prevent falls in older adults: an updated systematic review and meta-analysis. $\mathrm{Br}$ J Sports Med 2016.doi: 10.1136/bjsports-2016-096547. [Epub ahead of print 4 Oct 2016].
47. Slatkovska L, Alibhai SM, Beyene J, et al. Effect of 12 months of whole-body vibration therapy on bone density and structure in postmenopausal women: a randomized trial. Ann Intern Med 2011;155:668-79.

48. Bemben DA, Palmer IJ, Bemben MG, et al. Effects of combined whole-body vibration and resistance training on muscular strength and bone metabolism in postmenopausal women. Bone 2010;47:650-6.

49. Torvinen $\mathrm{S}$, Kannus $\mathrm{P}$, Sievänen $\mathrm{H}$, et al. Effect of 8-month vertical whole body vibration on bone, muscle performance, and body balance: a randomized controlled study. J Bone Miner Res 2003;18:876-84. 